\title{
DIFFERENTIABLE FUNCTIONS AND ROUGH NORMS ON BANACH SPACES
}

\author{
E. B. LEACH AND J. H. M. WHITFIELD
}

Abstract. The main result is that if $X$ is a real Banach space, such that the density character of $X^{*}$ is greater than that of $X$, then there does not exist any real-valued Fréchet differentiable function on $X$ with bounded nonempty support.

1. Introduction. The main purpose of this paper is to prove the theorems stated in [6], which give some fairly general types of Banach space on which there does not exist any real-valued Fréchet differentiable function with bounded nonempty support. Kurzweil [3] proved this for the spaces $C[0,1]$ and $l_{1}$ for continuously differentiable functions. Bonic and Frampton [1] removed the continuity condition. Restrepo [4], [5] and Kadec [2] proved that if $X^{*}$ has density character greater than that of $X$ (dens $X^{*}>$ dens $X$ ), there does not exist a Fréchet-smooth norm for $X$. Leduc [7] has proved that if dens $X^{*}>$ dens $X$, then there does not exist any continuously Fréchet differentiable funcrion on $X$ with bounded nonempty support. His proof is simpler than the proof of the stronger result in this paper. This paper will obtain the Kurzweil-Bonic-Frampton conclusion, using the Kadec-Restrepo hypothesis.

The main theorem is a trivial consequence of two theorems (2.1 and 3.2) proved below. The first of these states that if dens $X^{*}>$ dens $X$, then there is an equivalent "rough" norm for $X$, in a sense which will be made precise below. Theorem 3.2 states that if any equivalent norm for $X$ is rough, then there does not exist any Fréchet differentiable function on $X$ with bounded nonempty support. The method of proof of Theorem 3.2 is similar to that of Kurzweil [3], who used essentially the fact that the conventional norms for $C[0,1]$ and for $l_{1}$ are rough. The proof of Theorem 2.1 is a construction of a new norm $\sigma$, for which the unit ball is an intersection of half-spaces described by uniformly separated functionals from $X^{*}$.

Presented in part to the Society, September 3, 1965 under the title Differentiable functions with bounded non-empty support on Banach spaces; received by the editors January 18, 1971 and, in revised form, August 30, 1971.

AMS 1970 subject classifications. Primary 58C20, 46B05; Secondary 41A65.

Kev words and phrases. Fréchet differential, Gâteaux differential, one-sided Gâteaux differential, uniformly discontinuous, rough norm, density character, large set, bounded nonempty support. 
2. In this section we will prove that there is a "rough" norm of $X$, provided $X^{*}$ is strictly denser than $X$. First we define our meanings for differentiability. Let $X$ and $Y$ be Banach spaces with norms $\rho$ and $\sigma$ respectively; let $A$ and $B$ be open subsets, respectively, of $X$ and $Y$. A function $f: A \rightarrow B$ has Fréchet differential $f^{\prime} x$ at $x \in A$, if $\left(f^{\prime} x\right): X \rightarrow Y$ is a bounded linear transformation with the property that for every $\varepsilon>0$, there exists $\delta>0$, such that $\sigma\left(f(x+u)-f x-\left(f^{\prime} x\right) u\right) \leqq \varepsilon^{\cdot}(\rho u)$, whenever $\rho u<\delta$. A one-sided Gâteaux differential of $f$ at $x$ is a function $\left(f^{\prime} x\right): X \rightarrow Y$, possibly nonlinear, such that

$$
\left(f^{\prime} x\right) u=\lim _{t \rightarrow 0+} \frac{f(x+t \cdot u)-f x}{t}, \text { for each } u \in X .
$$

If the one-sided Gâteaux differential $f^{\prime} x$ is linear it is a Gâteaux differential.

The norm of $X$ always has a one-sided Gâteaux differential because the difference quotient is an increasing function of $t$, for $t \neq 0$. We will frequently use the fundamental and elementary inequality implied by this:

$$
\rho(x+t \cdot u) \geqq \rho x+t \cdot\left(\rho^{\prime} x\right) u, \text { for all } t, x \text { and } u \text {. }
$$

The density character of a Banach space $X$, denoted dens $X$, is the minimum cardinal number of a dense subset of $X$, or equivalently, it is the maximum cardinal number of a discrete subset of $X$. Any set $A$ will be called large (with respect to $X$ ), if card $A>\operatorname{dens} X$; otherwise $A$ will be called small.

The norm $\rho$ of $X$ has a uniformly discontinuous differential $\rho^{\prime}$ (as a one-sided Gâteaux differential), if there exists $\varepsilon>0$, such that for every $x \in X$ and $\eta>0$, there exist $x_{1}, x_{2}$ and $u$ in $X$, such that $\rho\left(x_{1}-x\right)<\eta$, $\rho\left(x_{2}-x\right)<\eta, \rho u=1$ and $\left(\rho^{\prime} x_{2}-\rho^{\prime} x_{1}\right) u \geqq \varepsilon$. This property is valid for $C[0,1]$ and for $l_{1}$, with $\varepsilon=2$. (We will sometimes use the adjective "rough" to mean the property described here. In general "rough", like "smooth", should be a flexible term to fit a given situation.)

The following is the same as Theorem 2 of [6], stated there without proof.

2.1. Theorem. If $X$ is a Banach space, such that dens $X<\operatorname{dens} X^{*}$, there is an equivalent norm $\sigma$ for $X$, such that $\sigma^{\prime}$ is uniformly discontinuous.

Proof. Let $E_{1}$ be a subset of $X^{*}$, chosen to be maximal subject to:

(i) $E_{1}$ contains the origin of $X^{*}$.

(ii) If $\alpha \in E_{1}$, then $-\alpha \in E_{1}$.

(iii) If $\alpha \neq \beta$ in $E_{1}$, then $\rho^{*}(\beta-\alpha)>1$ (where $\rho^{*}$ is the norm on $X^{*}$ ).

Evidently $E_{1}$ is also maximal subject only to (iii). For any real number $a$, let $E_{u}=\left\{a \cdot \alpha: \alpha \in E_{1}\right\}$. Since $\bigcup_{n=1}^{\infty} E_{1 / n}$ is dense, card $E_{1}=$ dens $X^{*}>$ dens $X$, that is, $E_{1}$ is large. There is an integer $n$, such that $E_{1}$ intersects 
the open ball of radius $n$ about the origin of $X^{*}$ in a large set. Then $E_{1 / 2}$ intersects every open ball of radius $n+\frac{1}{2}$ in a large set, and so $E_{1 / 4(2 n+1)}$ intersects every open ball of radius $\frac{1}{4}$ in a large set. The assertion about $E_{1 / 2}$ is the only one that is not obvious. To see this, consider any open ball of radius $n+\frac{1}{2}$ and center at $\gamma$. For each $\alpha \in E_{1}$, we may choose $\beta \in E_{1 / 2}$, so that $\rho^{*}(\alpha+\gamma-\beta) \leqq \frac{1}{2}$. This determines a one-to-one correspondence between $E_{1}$ and a subset of $E_{1 / 2}$; points of $E_{1}$ in the open ball of radius $n$ about the origin correspond to points of $E_{1 / 2}$ in the given ball of radius $n+\frac{1}{2}$, which points thus form a large set.

Let $F=\left\{\alpha: \alpha \in E_{1 / 4(2 n+1)}\right.$ and $\left.\rho^{*} \chi \leqq 1\right\}$. The norm $\sigma$ will be defined for any $x \in X$ by:

$$
\sigma x=\inf \{t: \alpha x \leqq t \text { for all except a small set of } \alpha \text { 's in } F\} .
$$

It is easy to see that $\sigma$ is a norm for $X$, and that $\sigma x \leqq \rho x$. Also, for any $x \in X,\{\alpha: \alpha \in F$ and $\alpha x \leqq \sigma x\}=\bigcap_{n=1}^{\infty}\{\alpha: \alpha \in F$ and $\alpha x \leqq \sigma x+1 / n\}$, so that $\alpha x \leqq \sigma x$ for all except a small set of $\alpha$ 's in $F$. For any $x$, let $x^{*}$ be a linear tangent functional at $x$, with $\rho^{*} x^{*}=1 . F$ intersects the open ball of radius $\frac{1}{4}$ about $\frac{3}{4} x^{*}$ in a large set, and $\alpha x>\frac{1}{2}(\rho x)$ for all $\alpha$ in this set, so that $\sigma x \geqq \frac{1}{2}(\rho x)$. Therefore $\sigma$ is equivalent to $\rho$.

The revised dual norm $\sigma^{*}$ satisfies $\sigma^{*} \alpha \geqq \rho^{*} \alpha$, so for all $\alpha \neq \beta$ in $F$, $\sigma^{*}(\beta-x)>1 / 4(2 n+1)$. But possibly $\sigma^{*} x>1$ for some $\alpha \in F$. Let $G$ be the intersection of $F$ with the closed unit ball about the origin under the $\sigma^{*}$ norm. We will show that $G$ contains all except a small subset of $F$. For this, let $D$ be a dense subset of $X$, with card $D=\operatorname{dens} X$. If $\sigma^{*} \alpha>1$, there exists $x \in D$, such that $\alpha x>\sigma x$; but for each $x$, this occurs only for a small set of $\alpha$ 's in $F$. So $\left\{\alpha: \alpha \in F\right.$ and $\left.\sigma^{*} \alpha>1\right\}=\bigcup_{x \in D}\{\alpha: \alpha \in F$ and $\alpha x>\sigma x\}$, and the cardinality of this union is no greater than (dens $X)^{2}=$ dens $X$.

We will now show that $\sigma^{\prime}$ satisfies the uniform discontinuity condition, with $\varepsilon=1 / 8(2 n+1)$. For any $x \in X$ and $\eta>0$ (we will assume that $\eta<$ $1 / 8(2 n+1)$ ), choose $\alpha \neq \beta$ in $G$, such that $\alpha x>\sigma x-\eta^{2} / 2$ and $\beta x>\sigma x-\eta^{2} / 2$, noting that $\alpha$ and $\beta$ may be chosen from a large subset of $G$. Then choose $u \in X$, such that $\sigma u=1$ and $(\beta-\alpha) u>1 / 4(2 n+1)$. Then if $t>0$, $\sigma(x+t \cdot u) \geqq \beta(x+t \cdot u)>\sigma x+t \cdot \beta u-\eta^{2} / 2$. From $(1)$,

$$
\sigma x \geqq \sigma(x+t \cdot u)-t \cdot\left(\left(\sigma^{\prime}(x+t \cdot u)\right) u\right) ;
$$

so on adding, transposing and dividing by $t,\left(\sigma^{\prime}(x+t \cdot u)\right) u \geqq(\beta u)-\eta^{2} / 2 t$, for $t>0$. Similarly, $-\left(\sigma^{\prime}(x-t \cdot u)\right) u \geqq-\alpha u-\eta^{2} / 2 t$, and adding, we have for any $t>0$ :

$$
\left(\sigma^{\prime}(x+t \cdot u)\right) u-\left(\sigma^{\prime}(x-t \cdot u)\right) u \geqq 1 / 4(2 n+1)-\eta^{2} / t .
$$

Now choose $t<\eta$, such that $\eta^{2} / t<1 / 8(2 n+1)$, which is possible because $\eta<1 / 8(2 n+1)$, and we obtain the uniform discontinuity condition with $x_{1}=x-t \cdot u$ and $x_{2}=x+t \cdot u$. 
The following lemma gives a property equivalent to uniform discontinuity of $\rho^{\prime}$ (although the value of $\varepsilon$ may change). The reverse implication can be proved by a method similar to the latter part of the proof of Theorem 2.1. This property is the key property needed to prove Lemma 3.1 .

2.2. Lemma. Let $X$ be a Banuch space with norm $\rho$, such that $\rho^{\prime}$ is uniformly discontinuous. For every $x \in X$, and $\eta>0$, there exists $v \in X$, such that $\rho v<2$ and $\rho(x+t \cdot v)>\rho x+\varepsilon \cdot|t| / 2-\eta$, where $\varepsilon$ is any number satisfying the uniform discontinuity condition and $|t| \leqq \rho x$.

Proof. Given $x$ and $\eta_{1}>0$, choose $x_{1}, x_{2}$ and $u$, using uniform discontinuity of $\rho^{\prime}$, so that $\rho\left(x_{1}-x\right)<\eta / 4, \rho\left(x_{2}-x\right)<r_{i} / 4, \rho u=1$ and $\left(\rho^{\prime} x_{2}-\rho^{\prime} x_{1}\right) u \geqq \varepsilon$. Let $v=u-\left(\left(\rho^{\prime} x_{1}+\rho^{\prime} x_{2}\right) u /(2 \cdot \rho x)\right) \cdot x$, so that $0<\rho v<2$. Now look at $x+t \cdot v$, when $0 \leqq t \leqq \rho x$ :

$x+t \cdot v=s \cdot x+t \cdot u$

$$
\begin{aligned}
& \quad\left(\text { where } s=1-\frac{t \cdot\left(\rho^{\prime} x_{1}+\rho^{\prime} x_{2}\right) u}{2 \cdot \rho x}, \text { and } 0<s<2\right) \\
& =s \cdot\left(x_{2}+\frac{t}{s} \cdot u\right)+s \cdot\left(x-x_{2}\right) .
\end{aligned}
$$

Then, using (1):

$$
\begin{aligned}
\rho(x+t \cdot v) & >s \cdot\left(\rho x_{2}+t / s \cdot\left(p^{\prime} x_{2}\right) u\right)-s \cdot \eta_{i}^{\prime 4} \\
& >s \cdot(\rho x)+t \cdot\left(\rho^{\prime} x_{2}\right) u-s \cdot \eta / 2 \\
& =\rho x+t / 2 \cdot\left(\rho^{\prime} x_{2}-\rho^{\prime} x_{1}\right) u-s \cdot \eta / 2 \\
& >\rho x+t \cdot \varepsilon / 2-\eta .
\end{aligned}
$$

If $-\rho x \leqq t<0$, we proceed in similar fashion, but refer to $x_{1}$ instead of $x_{2}$ :

$$
\begin{aligned}
\rho(x+t \cdot v) & >s \cdot(\rho x)+t \cdot\left(\rho^{\prime} x_{1}\right) u-s \cdot \eta / 2 \\
& =\rho x+(-t / 2) \cdot\left(\rho^{\prime} x_{2}-\rho^{\prime} x_{1}\right) u-s \cdot \eta / 2 \\
& >\rho x+|t| \cdot \varepsilon \varepsilon_{i}^{\prime 2}-\eta,
\end{aligned}
$$

which again is the desired inequality.

3. The main theorem (3.2) of this section is essentially Theorem 1 of [6], and in conjunction with Theorem 2.1 implies the main result (Theorem 3.3) of this paper.

3.1. Lemma. Suppose a Banach space $X$ has a norm $\rho$ with uniformly discontinuous differential $\rho '$. If $f$ is a real-valued Fréchet differentiable function on $X$ and $f 0=0$, then there exists $x \in X$, such that $1 \leqq \rho x<2$ and $f x \leqq \rho x$. 
Proof. We choose a sequence $\left\{x_{n}\right\}$ by induction to satisfy $x_{0}=0$ and:

(1) $f x_{n}-\rho x_{n} \leqq 0$,

(2) $\rho\left(x_{n+1}-x_{n}\right) \leqq 1$,

(3) $\rho x_{n+1} \geqq \rho x_{n}+(\varepsilon / 8) \cdot \rho\left(x_{n+1}-x_{n}\right)$,

(4) $\rho\left(x_{n+1}-x_{n}\right) \geqq \frac{1}{2} \cdot M_{n}$, where

$$
M_{n}=\sup \left\{\rho\left(y-x_{n}\right): y \in X \text { and } y=x_{n+1} \text { satisfies (1), (2) and (3) }\right\} .
$$

The number $\varepsilon$ is determined by the uniform discontinuity condition. In choosing $\left\{x_{n}\right\}$, one could always choose $x_{n+1}=x_{n}$, to satisfy (1), (2) and (3). The 4th condition requires a nontrivial choice of $x_{n+1}$, if possible.

We will show that for some $n, \rho x_{n} \geqq 1$. If not $\left\{\rho x_{n}\right\}$ is a Cauchy sequence, since it is increasing. Also by (3), if $m>n$,

$$
\begin{aligned}
\rho\left(x_{m}-x_{n}\right) & \leqq \rho\left(x_{m}-x_{m-1}\right)+\cdots+\rho\left(x_{n+1}-x_{n}\right) \\
& \leqq(8 / \varepsilon) \cdot\left[\left(\rho x_{m}-\rho x_{m-1}\right)+\cdots+\left(\rho x_{n+1}-\rho x_{n}\right)\right] \\
& =(8 / \varepsilon) \cdot\left(\rho x_{m}-\rho x_{n}\right),
\end{aligned}
$$

so that $\left\{x_{n}\right\}$ is a Cauchy sequence converging to $z \in X$, such that $\rho z \leqq 1$ and $f z-\rho z \leqq 0$.

Since $f$ is Fréchet differentiable at $z$, there exists $\delta>0$, such that $f(z+u)-f z-\left(f^{\prime} z\right) u \leqq \varepsilon \cdot(\rho u) / 8$, provided $\rho u<\delta$. We may also assume that $\delta \leqq 1$ and $\delta \leqq 2 \cdot(\rho z)$. By Theorem 2.2 , there exists $v$, such that $\rho v<2$ and $\rho(z+t \cdot v)>\rho z+\varepsilon \cdot|t| / 2-\varepsilon \cdot \delta / 8$, whenever $|t| \leqq \rho z$. Choosing $t= \pm \delta / 2$ depending on the sign of $\left(f^{\prime} z\right) v$ we have

$$
\rho(z+t \cdot v)>\rho z+\varepsilon \cdot \delta_{i} 8>\rho z+\varepsilon \cdot \rho(t \cdot v) / 8
$$

and

$$
f(z+t \cdot v)<f z+\varepsilon \cdot \rho(t \cdot v) / 8,
$$

from which we obtain the inequalities:

(1') $f(z+t \cdot v)-\rho(z+t \cdot v) \leqq 0$,

(2') $\rho(z+t \cdot v-z)<1$,

(3') $\rho(z+t \cdot v)-\rho z>\left(\varepsilon_{1}^{\prime} 8\right) \cdot \rho(t \cdot v)$.

Because $\rho$ and $f$ are continuous at $z$, we see that $x_{n+1}=z+t \cdot v$ satisfies (1), (2) and (3) for large $n$, so that $M_{n} \geqq \rho\left(z+t \cdot v-x_{n}\right)$. But since $\rho(z+t \cdot v-z)>\varepsilon \cdot \delta / 8, M_{n}>\varepsilon \cdot \delta / 8$, for all large $n, 8 \rho\left(x_{n+1}-x_{n}\right)>\varepsilon \cdot \delta / 16$ for all large $n$ by condition (4), contradicting convergence of $\left\{x_{n}\right\}$. So $\rho x_{n} \geqq 1$ for some $n$, and the smallest such $n$ gives rise to the vector $x=x_{n}$ satisfying the requirements of the lemma.

THEOREM 3.2. If $X$ has an equivalent norm $\rho$ such that $\rho^{\prime}$ is uniformly discontinuous, there does not exist a Fréchet differentiable real-valued function $f$ on $X$, with bounded nonempty support. 
Proof. If such a function exists, there is a function $f$ satisfying $f 0=2$ and $f x=0$, whenever $\rho x \geqq 1$. Let $g x=2-f x$. Then $g x-\rho x>0$, whenever $1 \leqq \rho x<2$, and so we obtain a violation of Lemma 3.1.

Theorems 2.1 and 3.2 can now be easily composed to give the main theorem:

THEOREM 3.3. If $X$ is a real Banach space, and if dens $X^{*}>\operatorname{dens} X$, then there does not exist any real-valued Fréchet differentiable function on $X$ with bounded nonempty support.

4. Consider the following conditions that might be satisfied by a real Banach space $X$ :

(i) Existence of a Fréchet-smooth norm for $X$ (note that Fréchet differentiability implies continuity of the differential of the norm at any point).

(ii) Existence of a real-valued continuously Fréchet differentiable function on $X$ with bounded nonempty support.

(iii) Existence of a real-valued Fréchet differentiable function on $X$ with bounded nonempty support.

If $X$ is separable, each of these is equivalent to the separability of $X^{*}$, for if $X^{*}$ is separable, Restrepo [5] and Kadec [2] have displayed a Fréchet-smooth norm, and if $X^{*}$ is not separable, our Theorem 3.2 applies. It is not known in general whether (i), (ii) and (iii) are equivalent, nor whether there is a simple equivalent condition in terms of density or rough norms. The condition dens $X=$ dens $X^{*}$ is necessary, but not sufficient, for one could take the cartesian product $l_{1} \times H$, when $H$ is a Hilbert space with dens $H \geqq \operatorname{dens} l_{1}^{*}$, and the sum of the component norms in $l_{1}$ and in $H$ gives a rough norm, so that Theorem 3.2 applies.

In the realm of reasonable conjecture, one could offer the following conditions:

(a) There does not exist a "rough" norm for $X$.

(b) There does not exist a "rough" norm for any subspace of $X$.

(c) There does not exist a "rough" norm for any separable subspace of $X$

(d) If $Y$ is a subspace of $X$, dens $Y=$ dens $Y^{*}$.

(e) If $Y$ is a separable subspace of $X, Y^{*}$ is separable.

Each of these conditions is necessary for any of the conditions (i), (ii) or (iii). Also, (b), (c), (d) and (e) are equivalent. To see this, note that failure of any of them implies, by Theorem 2.1, that there is a subspace $Y$ of $X$ on which there is an equivalent "rough" norm. The uniform discontinuity conditions can be used to choose a separable subspace $Z$ of $Y$ on which there is an equally rough norm, and since there does not exist 
a Fréchet-smooth norm for $Z$, by the Restrepo-Kadec theory, $Z^{*}$ is not separable.

We close with a final question. We have just noted that if $X$ has a subspace $Y$, such that dens $Y<$ dens $Y^{*}$, then $X$ has a separable subspace $Z$, such that $Z^{*}$ is not separable. Question: if dens $Y<$ dens $Y^{*}$, then for every infinite cardinal number $\alpha<$ dens $Y$, is there a subspace $Z$ of $X$, such that $\alpha=$ dens $Z<$ dens $Z^{*}$ ?

\section{REFERENCES}

1. R. Bonic and J. Frampton, Differentiable functions on certain Banach spaces, Bull. Amer. Math. Soc. 71 (1965), 393-395. MR 30 \#2310.

2. M. I. Kadec, Conditions for the differentiability of a norm in a Banach space, Uspehi Mat. Nauk 20 (1965), no. 3 (123), 183-187. (Russian) MR 32 \#2883.

3. J. Kurzweil, On approximation in real Banach spaces, Studia Math. 14 (1954), 214 231. MR 16, 932.

4. G. Restrepo, Differentiable norms in Banach spaces, Bull. Amer. Math. Soc. 70 (1964), 413-414. MR 28 \#4338.

5. —, Differentiable norms, Bol. Soc. Mat. Mexicana (2) 10 (1965), 47-55. MR 35 \#5901.

6. J. H. M. Whitfield, Differentiable functions with bounded nonempty support on Banach spaces, Bull. Amer. Math. Soc. 72 (1966), 145-146. MR 32 \#1547.

7. M. Leduc, Densité de certaines familles d'hyperplans tangents, C.R. Acad. Sci. Paris Sér. A 270 (1970), 326-328.

Department of Mathematics, Case Western Reserve University, Cleveland, OHIO 44106

Department of Mathematics, Lakehead University, Thunder Bay, Canada

Current address (J. H. M. Whitfield): Department of Mathematics, University of Washington, Seattle, Washington 98105 